\title{
Enseñar física a través del teatro
}

\section{Physics teaching through theater}

\author{
José Joaquín García-García ; Arley Fabio Ossa-Montoya ${ }^{2}$; Edilma Rentería-Rodríguez ${ }^{3}$ \\ 'Lic. en Biología y Química, M.Sc., Ph.D. Universidad de Antioquia, Facultad de Educación. Medellín - Antioquia, Colombia; e-mail: joaquin.garcia@udea.edu. \\ co; (1)https://orcid.org/0000-0002-5009-7942 \\ ²Lic. en Educación Física, M.Sc., Ph.D. Universidad de Antioquia, Facultad de Educación. Medellín - Antioquia, Colombia; e-mail: arley.ossa@udea.edu.co; \\ (Dhttps://orcid.org/0000-0002-0171-0317 \\ ${ }^{3}$ Lic. en Matemáticas y Física, M.Sc. Universidad de Antioquia, Facultad de Educación, Medellín - Antioquia, Colombia; e-mail: edilmarenteria@yahoo.es; \\ (Dhttps://orcid.org/0000-0003-1831-4502
}

Cómo citar: García-García, J.J.; Ossa-Montoya, A.F.; Rentería-Rodríguez, E. 2020. Enseñar física a través del teatro. Rev. U.D.C.A Act. \& Div. Cient. 23(2):e1139. http://doi.org/10.31910/rudca.v23.n2.2020.1139

Artículo de acceso abierto publicado por Revista U.D.C.A Actualidad \& Divulgación Científica, bajo una licencia Creative Commons CC BY-NC 4.0

Publicación oficial de la Universidad de Ciencias Aplicadas y Ambientales U.D.C.A, Institución de Educación Superior Acreditada de Alta Calidad por el Ministerio de Educación Nacional.

Recibido: Abril 12 de 2019 Aceptado: Octubre 15 de 2020 Editado por: Ingeborg Zenner de Polanía

\section{RESUMEN}

El problema de investigación en este trabajo es articular el arte del teatro, su pasión y su estética, a la formación científica, para pensar, sentir y disfrutar el mundo. El objetivo de este trabajo fue determinar cómo valoran los estudiantes de educación media y docentes en formación, el uso de la obra "Galileo Galilei”, para la enseñanza de la Física clásica. Esta investigación es interpretativa, con un diseño cualitativo de casos múltiples. Para la recolección y análisis de información, se usaron las técnicas de grupo focal y de análisis del discurso. Los resultados mostraron un aprendizaje más dinámico y divertido, cambio en las concepciones sobre la naturaleza de las ciencias y mejora en las relaciones sociales.

Palabras clave: Física; Teatro; Galileo Galilei; Didáctica; Gestión emocional.

\section{ABSTRACT}

The research problem in this work is to articulate the art of the theater with his passion and aesthetic, to a scientific formation, for thinking, feeling and enjoying the world. The objective of this research was to determine how the students of middle education and preservice teacher, value the use of the "Galileo Galilei" play, to teach classical physics. This research is interpretive, and qualitative design, with multiple cases. For the collection and analysis of information, the focus group and discourse analysis techniques were used. The results showed a more dynamic and fun learning, change in the conceptions about the nature of the sciences, and improve in social relations.

Keywords: Physics; Theater; Galileo Galilei; Didactic; Emotional management. 


\section{INTRODUCCIÓN}

Las prácticas educativas, se basan en la transmisión, la repetición y la memorización de contenidos sin contexto (García, 2014), pruebas objetivas y adquisición de competencias laborales, que no contribuyen a la formación integral de los sujetos. Igualmente, se enseña separando el amor del conocer, haciendo a la escuela en un lugar para padecer y al recreo, en el único espacio divertido en ella (Waichman, 2008). Frente a esto, surge la idea de llevar a la razón sensible a la escuela.

La razón sensible reúne razón y sentimiento, conocimiento y afecto y pensamiento con pasión, tornando el saber en arte, al ofrecer lo bello del mundo y al responder a las inquietudes vitales del sujeto (Durand, 1993). Para la razón sensible, solo aquel que sabe sentir, querrá actuar para mejorar el mundo (Ortega \& Gasset, 1984; Maffesoli, 1997). Así, para ella, enseñar es cultivar mente, corazón e imaginación y obtener el compromiso afectivo y cognitivo del estudiante (Hodson, 2003). La razón sensible podría entrar al aula, incorporando en ella la vitalidad del arte. Este trabajo propone vincular el teatro a la educación científica, para hacer del giro cognitivo del estudiante, un giro dramático: emocional, cálido, familiar y accesible, que le genere profundas huellas neuronales (Youngs, 2013).

El teatro es un "arte total", involucra mente, cuerpo y espíritu y articula en él música, danza, plástica, literatura, audiovisuales, fotografía e, inclusive, a la historia, la biología, la lengua y la cultura. El teatro persigue, reflejar, reflexionar, intervenir, transformar y criticar la realidad, influyendo en el público para construir una mirada crítica y estética. El teatro es una ceremonia que involucra sentimientos, ideas, acciones y sentidos, en una experiencia viva y consciente, en presente. El teatro, como experiencia de transformación, se nutre de diálogos, retos y estímulos, en una práctica inmersiva y lúdica, convirtiéndose en una herramienta pedagógica potente, para generar aprendizajes amplios, activos, gratificantes y con interrogantes intelectuales, personales y sociales (García et al. 2019). El teatro es "Mimicry", un tipo de juego, que implica representación (Caillois, 1986) y, como juego, no existe sin la acción, es decir, llevar el teatro al aula es usar motivadamente la lúdica y el juego, con su humor, errores e incertidumbres, generando autogestión, libertad, protagonismo y creatividad, a través de una experiencia con sentido. Así, disfrutar sería sinónimo de aprender, espacio lúdico para descubrir el mundo de escuela y ser con actitud y acción lúdica de maestro.

En la antigua Grecia, los ciudadanos se educaban en el teatro y en la edad media, los niños ya participaban de él. En clase de ciencias, el teatro conecta el mundo de la vida con el de las teorías, comunicándolas de forma alternativa y ampliada, al develar su carácter complejo y su impacto cultural, humano, histórico, ético, moral, económico, social, tecnológico, multidisciplinar y político, además de mostrar sus procesos de creación argumentativos, dialogantes, críticos, imaginativos y subjetivos (Moreira, 2012; Braund, 2015). El teatro en clase de ciencias podría ayudar a modificar las concepciones absolutistas, dogmáticas, elitistas, positivistas y empiristas sobre la ciencia, inculcadas en la enseñanza tradicional (Solomon, 1989), favoreciendo la construcción de una visión constructivista sobre la misma (Alias et al. 2014).

El teatro enriquece el espacio del aula de ciencias, colocando al estudiante y a su actividad en el centro. Eso lo logra, al explicar el mundo con experiencias ampliadas, más allá del lenguaje verbal (Brook, 2015) y usar el conflicto entre posiciones teóricas diferentes, en un experimento mental (Ferri, 2007). Además, al implicar experiencias coordinadas y armónicas con patrones dinámicos (al ser un arte temporal) y la interacción cuerpo (sentidos y energía), mente (pensamientos y emociones) y espacio (contexto y situación), genera un saber corporizado con sentido (García et al. 2019).

En el teatro científico, se enmarca un experimento en un conflicto dramático, para exponer su contenido, sus procedimientos y sus resultados (López-Vázquez, 2008). Este teatro, incluye el montaje de obras, de monólogos o de dramas creativos, con temática científica (García et al. 2017; García \& Parada, 2017). Esta investigación pretende relacionar el uso del teatro científico con la enseñanza de la Física. En Australia e Inglaterra, se han usado, con este fin, dramas creativos, diseñados por los estudiantes (Martínez, 2017). El teatro, también se ha usado para presentar, criticar e improvisar respuestas sobre fenómenos físicos (Morse, 2015). Igualmente, se ha utilizado para representar corporalmente objetos, variables, interacciones, fenómenos y entidades Físicas, como luz, sonido, circuitos, voltaje, energía o gravedad (Scherr et al. 2013; Taskin-Can, 2013).

Blanco et al. (2015) diseñaron y usaron dos obras: "Un domingo cualquiera" y "Un enigma", para enseñar los conceptos de presión y de resistencia de los materiales y adquirir competencias comunicativas y lingüísticas, logrando mayor apertura mental y un aprendizaje consciente, práctico y a largo plazo en los estudiantes. Martínez (2017) propone una obra en la que se enfrenten las posiciones geocéntrica y heliocéntrica, basada en lo aprendido en talleres teóricos y prácticos, para lograr mayor creatividad, interacción social y un aprendizaje agradable y sin presiones. Alias et al. (2014) muestran que el montaje de la obra "Entropía el secreto de los magos", para enseñar los conceptos de masa, peso, gravedad, temperatura, presión, energía interna y entropía, permite aprender haciendo preguntas y desde la acción de los personajes con los contenidos. Cordero et al. (2017) generaron en estudiantes de Física actitudes académicas positivas de satisfacción, alegría, entusiasmo y confianza, usando la obra "Gran Teatro de la Física", que trataba temas de mecánica, electricidad, ondas y magnetismo. Este trabajo propone indagar sobre el valor y el impacto que le asignan los estudiantes el montaje de la obra "Galileo Galilei", de Bertold Brecht, sobre cuatro aspectos: los procesos de aprendizaje y de enseñanza de la Física, sus concepciones sobre la naturaleza de las ciencias, los aspectos socioemocionales y las dificultades que ellos encontraron. Esta obra muestra cómo Galileo presentó su teoría, la defendió de las teorías rivales, su condena al destierro y el triunfo de sus ideas. 


\section{MATERIALES Y MÉTODOS}

Esta investigación tiene un diseño cualitativo abierto de casos múltiples. En ella participaron 31 estudiantes de décimo grado de la asignatura de Física clásica, en la Institución Educativa San Luis Gonzaga, del municipio de Copacabana, en el departamento de Antioquia, en Colombia y 15 docentes en formación de séptimo nivel, de la Licenciatura en Educación Básica, con énfasis en Ciencias Naturales y Educación Ambiental, de la Facultad de Educación, de la Universidad de Antioquia, del curso de Didáctica de las Ciencias 1. Ambos grupos recibieron formación básica en técnicas de actuación y de montaje de una obra teatral. La totalidad del grupo de Licenciatura actuó y las tareas de dirección, elaboración de vestuario y escenografía, fueron compartidas. El grupo de décimo grado, de forma concertada y libre, integró comisiones de dirección, narración, libretos, escenografía, vestuario, maquillaje y actuación y cada estudiante construyó una propuesta sobre su personaje o su función. Los dos grupos montaron y presentaron la obra en el primer semestre académico del 2017.

Para recolectar la información, se usó la técnica de grupos focales. Además, con los estudiantes de educación media, se realizó una encuesta acerca de la comprensión alcanzada sobre la obra. Al grupo de Licenciatura, se le preguntó: ¿Cómo les pareció la experiencia? ¿Cómo se sintieron en su desarrollo? ¿Este tipo de trabajo se puede usar en la enseñanza de ciencia? ¿Sí? ¿No? ¿Por qué? ¿Qué aprendieron acerca de la ciencia y los científicos con este trabajo? ¿Qué les enseñó en lo personal y en lo social esta experiencia? ¿Qué dificultades tuvieron al realizar el montaje de la obra? ¿Qué podríamos decir como conclusión acerca del trabajo realizado? Al grupo de estudiantes de décimo grado se les preguntó: ¿Cuál fue el papel que cada uno desempeñó en la obra? ¿Qué les enseñó la obra en lo personal y en lo social? ¿Cómo fue el proceso de construcción de la obra? ¿Si ustedes tuvieran que escoger entre unas clases expositivas y una obra de teatro para aprender física que escogerían y por qué? ¿Qué impacto tuvo la realización de la obra de teatro en su aprendizaje? ¿Qué impacto creen ustedes que tuvo la obra de teatro en la institución educativa? ¿Cómo se sintieron en el montaje de la obra? La información obtenida, se analizó construyendo varias categorías acerca del impacto y la valoración del montaje teatral científico, en los aspectos a estudiar, ya mencionados. La categorización de las respuestas a la encuesta ofreció una panorámica sobre el nivel de comprensión alcanzado acerca de la obra. Ambos grupos firmaron un acta de consentimiento informado autorizando a usar la información recolectada con fines estrictamente investigativos.

\section{RESULTADOS Y DISCUSIÓN}

A partir de las afirmaciones realizadas por el grupo de Licenciatura surgieron las siguientes categorías, sobre el impacto y la valoración del montaje y la presentación de la obra Galileo Galilei, en la clase de Física: mejoramiento de los procesos de enseñanza y aprendizaje, cambios en los aspectos sociales y los emocionales, concepciones acerca de la naturaleza de la ciencia y los científicos, y dificultades en la experiencia (Cuadro 1).
En primer lugar, la gran mayoría de afirmaciones justifican la utilidad del teatro para el aprendizaje, enfatizando en que era más claro, con mayor potencial y adquirido de una forma alternativa, además, de tener un carácter extra cotidiano, nuevo, contextual y más profundo. En segundo lugar, sobre la enseñanza, los estudiantes afirman que el uso del teatro en clase de Física es eficaz para mejorar la comunicación y las interacciones sociales entre los estudiantes y entre ellos y el docente, así como las posibilidades de dichas interacciones. Estos resultados son similares a los obtenidos por Caro (2014), quien reportó cómo, luego de la dramatización de la obra "Galileo Galilei" y en el curso de su montaje, mejoraron las relaciones entre docentes y estudiantes, eliminando el rechazo a las preguntas y la indiferencia al diálogo e incorporando la amistad, el respeto mutuo, la escucha y la sorpresa, ante las propias capacidades. Además, dicho estudio mostró que el espacio pasó de ser el aula gobernada por el docente, a espacios abiertos y democráticos, planeados y dirigidos en común. Igualmente, los estudiantes de Licenciatura califican la experiencia como de valor, divertida, interesante y motivadora, llamándola vivencia, con un reto enriquecedor y transformador.

Respecto a las afirmaciones acerca de la naturaleza de la ciencia y los científicos, los estudiantes de Licenciatura reconocen que el saber y sus teorías son constructos humanos de carácter social e histórico y que tienen un gran impacto en la sociedad. Así, esta experiencia de teatro científico, contribuyó a que desmitificaran a la ciencia y a los científicos, mostrando su humanidad y sus esfuerzos por usar la razón y los instrumentos tecnológicos (como el telescopio), para ir más allá de lo superficial.

Finalmente, el grupo de Licenciatura, aunque afirma que mejora su capacidad de gestión emocional, sus vínculos sociales y el reconocimiento de sí mismo y del otro, también reconoce que se les dificulta el trabajo en grupo y la relación social inicial con sus compañeros, porque, regularmente, realizar un trabajo académico solitario, no colaborativo, ni coordinado.

Las afirmaciones del grupo de educación media permitieron construir las siguientes categorías, sobre el impacto del uso del teatro para enseñar Física: mejoramiento de los procesos de aprendizaje, cambios en sus concepciones sobre la naturaleza de las ciencias, mejoramiento en las interacciones sociales y dificultades para aprender Física, a través del teatro (Cuadro 2).

En primer lugar, este grupo afirmó que la experiencia les enfrentó a planear y representar actividades prácticas y les generó un aprendizaje más profundo y con mayor motivación, porque requiere de investigar, pero también más eficiente, fácil, rápido y entretenido. Esto coincide con el aprendizaje reportado, a través de una secuencia narrativa expresada en una historia, en otras investigaciones (McFadden, 2012; Sutton, 2012).

El grupo de educación media también afirma sobre la naturaleza de la ciencia y sus procesos, que en ella se modelan fenómenos, utilizando la experimentación y la tecnología. Esto muestra una concepción contemporánea de la ciencia, como modelización cognitiva de la realidad. 
Cuadro 1. Categorías surgidas del grupo focal de Licenciatura.

\begin{tabular}{|c|c|c|}
\hline Categoría & Subcategoría & Afirmaciones \\
\hline \multirow{5}{*}{$\begin{array}{l}\text { El teatro es útil } \\
\text { para enseñar }\end{array}$} & Mejora el aprendizaje & $\begin{array}{l}\text { Deja una huella marcada en el aprendizaje - Da mayor poder para explicar y entender - Se aprende } \\
\text { de manera más profunda que en los libros - Permite buscar otras estrategias para aprender - Aclara la } \\
\text { información - También se produce un aprendizaje a través de los sentidos - Permite la interiorización de } \\
\text { la ciencia, dándole más significado a la lectura - Es una manera más dinámica de aprender ciencia. }\end{array}$ \\
\hline & $\begin{array}{l}\text { Cualifica la } \\
\text { interacción en } \\
\text { el aula: mayores } \\
\text { posibilidades de } \\
\text { pensamiento y } \\
\text { acción }\end{array}$ & $\begin{array}{l}\text { Permite interactuar de forma diferente con los estudiantes - Se pueden concertar más las ideas, aclarar } \\
\text { las mías y las de los otros - Permite socializar el conocimiento - Posibilita interactuar con el ambiente y } \\
\text { salir del aula - Mejora la comunicación y la capacidad de dar mensajes de forma asertiva - Permite hacer y } \\
\text { sentir cierto tipo de acciones - Posibilita ser más creativos y participativos a los estudiantes. }\end{array}$ \\
\hline & $\begin{array}{l}\text { Hace interesante los } \\
\text { temas }\end{array}$ & $\begin{array}{l}\text { Los sujetos se involucran más en lo que aconteció - Se valora más el tipo de aprendizaje fundado en } \\
\text { la razón sensible - Hace entretenido el conocimiento y aumenta la motivación - Hace más ameno el } \\
\text { ambiente del aula al hablar de temas "aburridos" y abstractos - Fue una experiencia: agradable que gustó } \\
\text { mucho, emocionante, enriquecedora, transformadora, novedosa - Permite perder el miedo, cambiar y } \\
\text { probar algo nuevo - Un reto para hacer una adecuada interpretación. }\end{array}$ \\
\hline & Novedad & $\begin{array}{l}\text { Se sale de lo cotidiano - Aborda la ciencia desde enfoques nuevos y diferentes - Permite relacionar la } \\
\text { ciencia con la actualidad - Usa lo histórico y hace traducible y representable el conocimiento - Permite } \\
\text { adaptar y contextualizar el conocimiento. }\end{array}$ \\
\hline & Profundidad & $\begin{array}{l}\text { Toca investigar más - Obliga a buscar más fuentes -Invita a investigar sobre los experimentos, sus } \\
\text { motivaciones y resultados. }\end{array}$ \\
\hline \multirow{4}{*}{$\begin{array}{l}\text { El teatro mejora } \\
\text { las emociones } \\
\text { y las relaciones } \\
\text { sociales }\end{array}$} & $\begin{array}{l}\text { Relaciones sociales } \\
\text { en el aula }\end{array}$ & $\begin{array}{l}\text { Permite formar lazos familiares con los compañeros - Favorece el que se establezcan conexiones fuertes } \\
\text { con ellos - Hace muy importante el trabajo en grupo - Se genera apoyo para el otro y reconocimiento del } \\
\text { otro - Se experimenta otro nivel de diálogo. }\end{array}$ \\
\hline & Reconocer al otro & $\begin{array}{l}\text { Se logran ver las capacidades propias y las del otro - Permite ponerse en el papel del otro y entender su } \\
\text { situación. }\end{array}$ \\
\hline & $\begin{array}{l}\text { Consolidación de sí } \\
\text { mismo }\end{array}$ & $\begin{array}{l}\text { Aumenta la confianza en el otro y en sí mismo - Se vence el miedo al público - Se aprende a improvisar - } \\
\text { Mejora la responsabilidad porque demanda de un montaje que será presentado al público. }\end{array}$ \\
\hline & $\begin{array}{l}\text { Visión de la } \\
\text { humanidad }\end{array}$ & Proporciona una visión diferente de la humanidad - El teatro nos hace más humanos. \\
\hline \multirow{2}{*}{$\begin{array}{l}\text { Carácter de los } \\
\text { científicos }\end{array}$} & $\begin{array}{l}\text { El científico es } \\
\text { humano }\end{array}$ & $\begin{array}{l}\text { Es la definición humana del científico - El científico tiene miedos, temores, intereses y recursos - El } \\
\text { reconocimiento es esencial para el científico. }\end{array}$ \\
\hline & $\begin{array}{l}\text { Interacciones entre } \\
\text { científicos }\end{array}$ & $\begin{array}{l}\text { Permite explorar a los científicos de manera diferente - No es sólo un científico, sino muchos y hay que } \\
\text { darle a cada uno el crédito. }\end{array}$ \\
\hline \multirow{7}{*}{$\begin{array}{l}\text { Naturaleza de la } \\
\text { ciencia }\end{array}$} & $\begin{array}{l}\text { Ciencia: constructo } \\
\text { sociocultural }\end{array}$ & $\begin{array}{l}\text { Una teoría es un constructo social en el que intervienen muchos actores y condiciones socioculturales - } \\
\text { La ciencia es una construcción humana. }\end{array}$ \\
\hline & $\begin{array}{l}\text { Ciencia: } \\
\text { construcción } \\
\text { humana }\end{array}$ & La ciencia y el científico afrontan errores, triunfos, perdidas enfermedades, dolores y hasta la muerte. \\
\hline & $\begin{array}{l}\text { El impacto de la } \\
\text { ciencia }\end{array}$ & La ciencia tiene un gran impacto en la educación, en lo económico, lo político y lo social. \\
\hline & $\begin{array}{l}\text { Metodología } \\
\text { científica }\end{array}$ & $\begin{array}{l}\text { La visión superficial conduce a errores, se debe estudiar profundamente los fenómenos como lo hacía } \\
\text { Galileo - Se reconoce el papel de los instrumentos como el telescopio para generar teorías. }\end{array}$ \\
\hline & $\begin{array}{l}\text { Incorporación de la } \\
\text { historia de la ciencia }\end{array}$ & $\begin{array}{l}\text { Permite aprender mucho de la historia de la ciencia, es una manera diferente de leerla - Ofrece } \\
\text { conocimientos que no se podrían obtener de otra manera - Muestra la realidad de los acontecimientos de } \\
\text { forma profunda. }\end{array}$ \\
\hline & Sociales & $\begin{array}{l}\text { Poca costumbre de trabajar en grupo - Desconexión social inicial con los compañeros - Poca } \\
\text { coordinación para expresar ideas y plasmarlas en acciones. }\end{array}$ \\
\hline & Personales & Inexperiencia en el teatro - Poco tiempo. \\
\hline
\end{tabular}


Cuadro 2. Categorías surgidas del grupo focal de décimo grado.

\begin{tabular}{|c|c|c|}
\hline Categorías & Subcategorías & Afirmaciones \\
\hline \multirow[t]{3}{*}{ Aprendizaje } & $\begin{array}{l}\text { Favorece la modeli- } \\
\text { zación de actividades } \\
\text { prácticas }\end{array}$ & $\begin{array}{l}\text { La mayoría de las discusiones y preguntas se centraron en la manera de representar las actividades } \\
\text { prácticas: ¿Cómo realizaremos la actividad? ¿Qué materiales utilizamos? ¿Esto sí representa lo que } \\
\text { Galileo quiso decir? ¿El experimento sí concuerda con lo propuesto en la obra? ¿Cómo se construye } \\
\text { un telescopio? Además, se realizaron representaciones prácticas sobre: la explicación que da Galileo a } \\
\text { Andreas, para demostrar que la tierra gira alrededor del sol y de que él, no colgaría de cabeza durante } \\
\text { la noche. }\end{array}$ \\
\hline & $\begin{array}{l}\text { Más profundidad en el } \\
\text { aprendizaje }\end{array}$ & $\begin{array}{l}\text { En la presentación de una obra de teatro no nos podemos levantar hasta resolver las dudas, es necesa- } \\
\text { rio que se investigue para hacer el montaje. }\end{array}$ \\
\hline & Más motivación & $\begin{array}{l}\text { Aprender así es mucho más entretenido, siempre estamos prestando atención, contrario a lo que pasa } \\
\text { en una clase normal. La obra nos permite entender más fácil la Física, porque seguimos una secuen- } \\
\text { cia, una historia. La obra nos permite entender más rápido, es mucho más eficiente para la compren- } \\
\text { sión. }\end{array}$ \\
\hline \multirow{3}{*}{$\begin{array}{l}\text { Favorece las inter- } \\
\text { acciones sociales }\end{array}$} & $\begin{array}{l}\text { Favorece el trabajo en } \\
\text { equipo y el aprendizaje } \\
\text { colaborativo }\end{array}$ & $\begin{array}{l}\text { El trabajo en equipo es clave para hacer que actividades como esta funcionen, nos corregimos entre } \\
\text { nosotros, sentí el compañerismo cuando me ayudaron con mi vestuario. Durante la presentación de } \\
\text { la obra mis compañeros me dieron ánimo, siempre estábamos apoyándonos en lo que se necesitara, } \\
\text { como cambio de vestuario, logística, maquillaje, corrección de discurso. }\end{array}$ \\
\hline & $\begin{array}{l}\text { Favorece la confianza } \\
\text { en los demás }\end{array}$ & $\begin{array}{l}\text { El director de la obra fue muy bueno y a medida que fue avanzando se notó su liderazgo, todos } \\
\text { íbamos a su voz. }\end{array}$ \\
\hline & Mayor autonomía & $\begin{array}{l}\text { A medida que avanzamos fuimos trabajando sin acompañamiento docente. En el transcurso de la } \\
\text { obra se cambió de personaje, de acuerdo con las habilidades de cada estudiante. Cada estudiante } \\
\text { construyó su personaje y lo socializó con el resto del grupo. }\end{array}$ \\
\hline $\begin{array}{l}\text { Dificultades de la } \\
\text { experiencia }\end{array}$ & $\begin{array}{l}\text { Timidez- fallo en ex- } \\
\text { presión oral. Tiempo } \\
\text { insuficiente }\end{array}$ & $\begin{array}{l}\text { Al principio todo era juego, sin reglas, pero daba vergüenza introducirse en el papel. Se sentía que el } \\
\text { trabajo que estaban realizando no era importante. Hacer la obra requiere de mucho tiempo. Al prin- } \\
\text { cipio solo dos o tres compañeros tenían actitudes para hablar en público, a los demás nos daba pena } \\
\text { o no hablábamos fuerte }\end{array}$ \\
\hline
\end{tabular}

Este grupo también afirma que se mejoran el trabajo en equipo, la confianza, y el compañerismo, lo que genera el estado de ánimo ideal para lograr un aprendizaje colaborativo. Así, el montaje teatral, dinamizó la colaboración, estrechó los lazos de amistad y disminuyó la competencia con el otro, humanizando el aprendizaje. Ellos también afirman que ganaron autonomía, independencia y liderazgo, al abordar, de forma personal, la construcción del rol dentro de la obra (personaje o función), acorde con sus habilidades, lo que coincide por lo señalado en otra investigación (Lillard et al. 2011).

Los estudiantes de educación media, también reportan dificultades como: infravaloración inicial del teatro, poco tiempo para ensayar, timidez para hablar en público y con sus pares y vergüenza al interpretar el personaje, lo que concuerda con otros estudios (Navarro \& Mantovani, 2012). Dichas dificultades son las mismas que afronta un adolescente para construir pensamiento estético, establecer vínculos sociales duraderos y anteponer su voluntad a la vergüenza de ser o hacer, es decir, para encontrarse a sí mismo.

Las respuestas de los estudiantes de educación media en la encuesta generaron las categorías: ciencia dinámica con procesos y procedimientos propios, carácter histórico y sociocultural de la ciencia, ciencia como fenómeno sociopolítico atravesado por el poder (Cuadro 3).
En primer lugar, los estudiantes de educación media muestran comprensión del carácter dinámico no lineal e incierto de la ciencia, carácter contrario al absolutista y estático, que presenta la educación tradicional. Así, ellos afirman que en la experiencia experimentan dudas, contradicciones, diversos puntos de vista, controversias, contrariedades, incertidumbres y el uso del recurso a la evidencia. Igualmente, ellos afirman que comprender las diferencias entre la visión geocéntrica y la propuesta heliocéntrica, requiere del llevar a cabo procesos científicos, como la modelización experimental, las demostraciones, el uso de la lógica, la argumentación sencilla o el uso de teorías, para así hacer frente a la fe ciega y a las creencias, con sus mentiras inmutables. En segundo lugar, los estudiantes muestran que comprenden el carácter histórico, sociocultural y no absoluto de la ciencia, es decir, que ella está inmersa en una época y una cultura y que es influida, por sus costumbres, regímenes, instituciones, coyunturas sociales, creencias, valores, moral, ética, intereses, y nivel de desarrollo, aunque, a veces, no coincida con ellos.

Finalmente, los estudiantes logran una comprensión de la ciencia como fenómeno sociopolítico, argumentando que su ausencia refleja el desinterés del poder dictatorial por el pueblo al que no le importa su suerte, pueblo que muchas veces está conforme, creyendo en supuestas verdades, inclusive, cuando hay pruebas en su contra, poniendo la Fe por delante de la ciencia. Igualmente, ellos reconocen 
Cuadro 3. Respuestas del grupo de décimo grado a la encuesta.

\begin{tabular}{|c|c|c|}
\hline Interrogante & Categorías & Afirmaciones \\
\hline $\begin{array}{l}\text { ¿Cómo convenció } \\
\text { Galileo a Andreas } \\
\text { de que la tierra gira } \\
\text { alrededor del sol y } \\
\text { de que no colgaría } \\
\text { de cabeza durante la } \\
\text { noche? }\end{array}$ & $\begin{array}{l}\text { Procesos científicos: } \\
\text { Favorece la modelización } \\
\text { experimental } \\
\text { y el aprendizaje conceptual }\end{array}$ & $\begin{array}{l}\text { Con un experimento de que Andrea era la tierra y otro objeto el sol y lo rotó a } 180^{\circ} \\
\text { alrededor del "sol". Haciendo el ejemplo de por dónde sale el sol y por dónde se } \\
\text { esconde con entorno a la tierra, si fuera el sol el que girara. Al hacer ejercicio de } \\
\text { colocarlo en una silla y hacerlo girar alrededor de un sol improvisado, es decir, con } \\
\text { argumentos lógicos y sencillos. }\end{array}$ \\
\hline $\begin{array}{l}\text { ¿Qué argumentaba la } \\
\text { Iglesia en contra de } \\
\text { Galileo? }\end{array}$ & $\begin{array}{l}\text { Naturaleza sociocultural de la } \\
\text { ciencia: } \\
\text { Favorece la compresión de } \\
\text { la naturaleza de la ciencia: las } \\
\text { coyunturas sociales influyen la } \\
\text { construcción de la ciencia }\end{array}$ & $\begin{array}{l}\text { Todo lo que él decía era mentira solo porque iba en contra de la Iglesia. De que en la } \\
\text { biblia decían que la tierra era el centro del universo y si Dios decía eso, así era. Que la } \\
\text { tierra era el centro del universo ya que había sido creada por Dios y era absurdo pensar } \\
\text { que su mayor creación se (..) era un simple astro. Que no quería aceptar la verdad de } \\
\text { que Galileo estaba dando otro punto de vista. Que Galileo estaba diciendo cosas en } \\
\text { contra de la Iglesia y haciendo teorías erróneas según ellos. Que el centro de la tierra } \\
\text { era la Iglesia y por tanto la tierra es el centro del universo. Que la tierra era el centro del } \\
\text { universo porque la institución así lo decía. De que el centro del universo era la tierra y } \\
\text { que las teorías propuestas por Galileo iban en contra de Dios y sus creencias. }\end{array}$ \\
\hline $\begin{array}{l}\text { ¿Qué contra/ } \\
\text { argumentaba Galileo } \\
\text { en contra de la Iglesia? }\end{array}$ & $\begin{array}{l}\text { Naturaleza sociocultural de } \\
\text { la ciencia construcción de } \\
\text { la ciencia un proceso social } \\
\text { controversial y con diferentes } \\
\text { visiones }\end{array}$ & $\begin{array}{l}\text { Que se negaban a lo evidente, que efectivamente la tierra no era el centro del universo, } \\
\text { que el sol no giraba a su alrededor sino al contrario... (no legible). Que tenía que aceptar } \\
\text { la verdad porque sus pruebas eran inmutables. La Iglesia se negaba ante nuevas teorías } \\
\text { solo por no salir de su zona de confort. Que la Iglesia se negaba a ver otras teorías por } \\
\text { que la única teoría correcta debía ser la de ellos. Que se negaban a que las personas } \\
\text { conocieran otro punto de vista. }\end{array}$ \\
\hline \multirow{3}{*}{$\begin{array}{l}\text { ¿Porqué para el monje } \\
\text { los conocimientos } \\
\text { de Galileo eran } \\
\text { peligrosos? }\end{array}$} & $\begin{array}{l}\text { Naturaleza sociocultural de las } \\
\text { ciencias: construcción influida } \\
\text { por costumbres y creencias }\end{array}$ & $\begin{array}{l}\text { Porque iba en contra de él y sus creencias. Porque esas no eran las creencias de la Iglesia. } \\
\text { Porque se salían de las costumbres y conocimientos que se manejaban en aquel entonces. } \\
\text { Porque se salían de las costumbres y consentimientos que se manejaban en aquel } \\
\text { entonces. Porque iba en contra los principios de la Iglesia. } \\
\text { Porque iba en contra de su credo y hacía que el pueblo se revelara. }\end{array}$ \\
\hline & $\begin{array}{l}\text { Naturaleza sociopolítica de las } \\
\text { ciencias: poder, Intereses }\end{array}$ & $\begin{array}{l}\text { Porque la gente al entender los conocimientos de Galileo ponía en peligro la Iglesia y } \\
\text { que ellos eran el centro del universo. Galileo contradecía a la Iglesia y la Iglesia tenía } \\
\text { miedo de perder el poder. Ponían en riesgo el imperio católico. La gente abría su mente } \\
\text { y eso afectaba a la Iglesia. ponía en riesgo la credibilidad de lo que decía la Iglesia. La } \\
\text { Iglesia podría perder el poder sobre la sociedad. }\end{array}$ \\
\hline & $\begin{array}{l}\text { Naturaleza dinámica de las } \\
\text { ciencias }\end{array}$ & $\begin{array}{l}\text { Galileo podía cambiar completamente la imagen que las personas tenían del universo, } \\
\text { desafiando a la Iglesia corriendo el riesgo de ser asesinado. Podía despertar un } \\
\text { sentimiento de duda permanente sobre las masas, desprestigiando y poniendo en la } \\
\text { incertidumbre el poder de Dios y la autoridad eclesial, en tanto la gente empezaría a } \\
\text { creer y pensar en otras cosas. Porque describía otro conocimiento superior al de los } \\
\text { monjes, Galileo tuvo investigaciones más avanzadas que otros, aspecto que abría la } \\
\text { mente y eso afectaba a la Iglesia. }\end{array}$ \\
\hline $\begin{array}{l}\text { ¿Cómo era el } \\
\text { cielo descrito por } \\
\text { Aristóteles? }\end{array}$ & $\begin{array}{l}\text { Naturaleza histórica de la } \\
\text { ciencia: Se conoce su historia }\end{array}$ & $\begin{array}{l}\text { El universo es inmutable, y lleno de estrellas. Los planetas giraban alrededor de la tierra } \\
\text { estable, inmutable Infinito }\end{array}$ \\
\hline \multirow{2}{*}{$\begin{array}{l}\text { Explica la siguiente } \\
\text { expresión:-Que solo } \\
\text { quieren besar los pies } \\
\text { al Papa cuando pisotea } \\
\text { al pueblo }\end{array}$} & $\begin{array}{l}\text { Naturaleza sociopolítica de las } \\
\text { ciencias: autoridad }\end{array}$ & $\begin{array}{l}\text { Solo reconocen al Papa cuando los pisotea. El pueblo se arrodilla ante él. A quien más } \\
\text { adulas, más daño te puede hacer. Que solo ayudan al Papa y dejaban al pueblo al lado. } \\
\text { Obedecen al Papa, aunque a este no le importen sus creyentes. Que cuando el Papa } \\
\text { pisotea al pueblo, creen que hacen lo correcto y el pueblo se humilla. Es una denuncia } \\
\text { hacia el poder dictatorial de la Iglesia. Es un disgusto causado por el poder de la Iglesia. }\end{array}$ \\
\hline & $\begin{array}{l}\text { Naturaleza dinámica de las } \\
\text { ciencias: } \\
\text { necesidad de la prueba }\end{array}$ & $\begin{array}{l}\text { Solo creen en lo que dice el Papa, aunque haya pruebas de lo contrario. Porque sin } \\
\text { pruebas no hay fe. }\end{array}$ \\
\hline $\begin{array}{l}\text { Explica la expresión: } \\
\text { donde la fe reinó } \\
\text { durante mil años, } \\
\text { ahora reina la duda. }\end{array}$ & $\begin{array}{l}\text { Naturaleza dinámica de la } \\
\text { ciencia: } \\
\text { No es absoluta y necesita de } \\
\text { pruebas }\end{array}$ & $\begin{array}{l}\text { Ya es tiempo de dejarse llevar por la razón. El cambio de las creencias por la duda. La } \\
\text { era de los descubrimientos. Hacer referencia a la era de la ciencia. Que el ser humano se } \\
\text { cuestiona al pasar los años. La tierra de Galileo hizo dudar a algunos. La fe fue cambiada } \\
\text { por la duda. Porque sin pruebas se acaba la fe. Por qué ahora hay contradicciones sobre } \\
\text { lo que antes se creía. }\end{array}$ \\
\hline
\end{tabular}


que la ciencia puede poner en peligro al poder, su credibilidad y sus intereses, desprestigiándolo, al hacer que el pueblo salga de su zona de confort, dude y deje de obedecer ciegamente; se rebele, abra su mente y cambie su visión del mundo, usando la ciencia.

\section{Consideraciones finales}

El aprendizaje logrado usando el teatro, al parecer es extra/cotidiano, sin las repeticiones de la rutina, con elementos sorpresivos, alternativos e innovadores. Además, constituye un reto que requiere planear e investigar e, involucra, un máximo de atención y de participación. Este tipo de aprendizaje, al ser protagónico y en activo, empodera al estudiante con una participación "total" y contextual (cuerpo, mente, sentimiento, espacio) y se amplifica, porque desarrolla su creatividad, liderazgo, autonomía e independencia.

Los resultados, también permiten concluir que los estudiantes logran una comprensión más racional de la ciencia, involucrando la duda, la controversia, las demostraciones, el uso de la tecnología, la lógica, el debate, la argumentación, la experimentación, la modelización y la solución de problemas. Asimismo, ellos amplían su concepción sobre la ciencia y sus posibilidades, incluyendo sus aspectos históricos, culturales, sociales y políticos, con sus necesidades, preguntas, condiciones y límites. Igualmente, este montaje teatral científico permite entender a los estudiantes que la ciencia es un instrumento que libera al sujeto de prejuicios, de sujeciones y de falsas creencias, para que pueda hacerse participe, con pleno derecho de las sociedades democráticas y tome las mejores decisiones en ellas. Así, se confirma la superioridad del teatro para visibilizar las diversas dimensiones de la experiencia, llamada ciencia (Denzin, 1997).

Finalmente, los estudiantes, a través del montaje teatral científicos, mejoran sus competencias socioafectivas, optimizando la gestión emocional, el establecimiento de vínculos y la comunicación, usando el diálogo respetuoso, en el que es central la escucha. Es este tipo de diálogo en el que se permite el reconocimiento del otro y es necesario para construir paz en la democracia. Igualmente, el montaje teatral mejoró en los estudiantes la autonomía y la confianza para hablar en público y para ser los líderes de su aprendizaje.

Estos hallazgos muestran la pertinencia de incluir el teatro en las clases de ciencias en la educación media y en la formación de docentes (Calvo, 2011). Además, este trabajo es un llamado a docentes, investigadores, científicos y artistas, para crear formas alternativas, extra cotidianas y vitales para enseñar las ciencias, que involucren la pasión por el mundo y por la vida y que permitan la participación y el compromiso de estudiantes y docentes.

Conflicto de intereses: El artículo fue preparado y revisado con la participación de todos los autores, quienes declaramos que no existe conflicto de intereses que ponga en riesgo la validez de los resultados presentados. Financiación: Este artículo es parte de los resultados obtenidos en el proyecto de investigación "La razón sensible en la educación: las potencialidades de la representación semiótica teatral y del drama creativo para la educación en Ciencias" Código 2016-
11445, financiado por la Facultad de Educación de la Universidad de Antioquia y la Asociación Pequeño Teatro de Medellín.

\section{REFERENCIAS}

1. ALIAS, E.; PERALTA, M.D.; TOLEDO, S. 2014. Ciencia y teatro en la escuela primaria: dos lenguajes para mejorar la práctica docente. Facultad de ingeniería - Universidad Nacional de Mar del Plata. Jornadas Nacionales sobre Pedagogía de la Formación del Profesorado: Investigar las prácticas para mejorar la formación: metodologías y problemas".

2. BLANCO, A.; MARTÍNEZ, M.; GONZÁLEZ, S. 2015. Ciencia y teatro: una experiencia de teatro científico con alumnado de educación secundaria. Revista Iberoamericana de Educación. 69(3):81-92. https://doi.org/10.35362/rie693113

3. BRAUND, M. 2015. Drama and learning science an empty space. British educational research journal. 41(1):102-121.

4. BROOK, P. 2015. El Espacio Vacío. Barcelona, España: Península.

5. CAILLOIS, R. 1986. "Los juegos y los hombres”, FCE, México.

6. CALVO, M.A. 2011. Actividad de teatro científico como recurso en la formación de futuros profesores. Revista Textos 69:93-98.

7. CARO, L. 2014. Planteamiento didáctico en la clase de física a partir de las actividades totalidad abiertas y el teatro para formar espíritu científico en estudiantes de Galileo Galilei 400 años después de la conquista del universo. Disponible desde Internet en:

http://soda.ustadistancia.edu.co/enlinea/congreso/ Coloquio/MEMORIAS\%20COLOQUIO\%20PDF/ INTERCULTURALIDAD/ (con acceso 14/10/2018).

8. CORDERO, H.; LOMBARDI, G.; FUENMAYOR, E.; VERRILLI, D.; CROCE, N.; CONTRERAS, E. 2017. El teatro como estrategia movilizadora de emociones y actitudes hacia las clases de física. Investigações em Ensino de Ciências. 22(1):189-221.

9. DENZIN, N. 1997. Interpretive ethnography: Ethnographic practices for the 21 st century. Ed. Sage Publications (London). 352p.

10. DURAND, G. 1993. De la mitocrítica al mitoanálisis. Ed. Anthropos (España). 366p.

11. FERRI, A.J. 2007. Willing suspension of disbelief: Poetic faith in film. Lanham: Lexington Books. 103p. 
12. GARCÍA, J.J. 2014. La razón sensible como posibilidad para un nuevo tipo de educación. Educación y Ciencia. (Colombia). 17:69-78.

13. GARCÍA, J.J.; PARADA, N.J. 2017. La razón sensible en la educación científica: las potencialidades del teatro para la enseñanza de las ciencias. Zona Próxima. (Colombia). 26:114-139.

http://dx.doi.org/10.14482/zp.26.10204

14. GARCÍA, J.J.; PARADA, N.J.; OSSA, A.F. 2017. El drama creativo una herramienta para la formación cognitiva, afectiva, social y académica de estudiantes y docentes. Rev. Latinoamericana de Ciencias Sociales, Niñez y Juventud. (Colombia). 15(2):839-859. http://dx.doi.org/10.11600/1692715x.1520430082016

15. GARCÍA, J.J.; PARADA, N.J.; OSSA, A.F. 2019. Teatro para aprender, enseñar y curar. Siglo del Hombre Editores. Bogotá, Colombia.

16. HODSON, D. 2003. Time for action: science education for an alternative future international. Internal J. of Science Education. (Australia). 25(6):645-670. https://doi.org/10.1080/09500690305021

17. LILLARD, A.S.; PINKHAM, A.; SMITH, E.D. 2011. Pretend play and cognitive development. En: Goswami, U. Ed. Handbook of cognitive development. Ed. Blackwell (London). p.285-311.

18. LÓPEZ-VÁZQUEZ, A.R. 2008. Aprender a ver teatro. Empezar a hacer teatro. Revista de Literatura-Especial teatro. 233:75-81.

19. MAFFESOLI, M. 1997. Elogio de la Razón Sensible: Una visión intuitiva del mundo contemporáneo. Ed. Paidos (España). 270p.

20. MARTÍNEZ, G.F. 2017. La dramatización como recurso para la enseñanza de la Física en el Bachillerato. Somos o no el centro del universo. Master universitario de formación del profesorado. Universidad de Granada.

21. MCFADDEN, B.L. 2012. Integrating theatre arts techniques into your curriculum. Kappa Delta Pi Record. (U.S). 48(2):87-91. https://doi.org/10.1080/00228958.2012.680388
22. MOREIRA, L.M. 2012. Oxigênio: uma abordagem filosófica visando discussões acerca da educação em ciências. parte 1: poder e ambição. Ciência y Educação. 18(4):803-818. http://dx.doi.org/10.1590/S1516-73132012000400005

23. MORSE, R.A. 2015. Commedia dell'Arte as a Metaphor for the Art of Teaching. The Physics Teacher. 53(392):1-2. https://doi.org/10.1119/1.4931002

24. NAVARRO, R.; MANTOVANI, A. 2012. El juego dramático de 5 a 9 años. Ed. Octaedro. (España). 29p.

25. ORTEGA, Y.; GASSET, J. 1984. ¿Qué es el conocimiento? Ed. Alianza. (España). 184p.

26. SCHERR, E.; CLOSE, G.; CLOSE, E.; FLOOD, J.; MCKAGAN, S.; ROBERTSON, D.; SEELEY, L.; WITTMANN, M.; VOKOS, S. 2013. Negotiating energy dynamics through embodied action in a materially structured environment. Physical Review Physics Education Research. 9:1-18. https://doi.org/10.1103/PhysRevSTPER.9.020105

27. SOLOMON, J. 1989. The Trial of Galileo. En: Herget, D. The History and Philosophy of Science in Science Teaching. Proceedings of the First International Conference. Conference in Houston, Texas, EE.UU. 36p.

28. SUTTON, P. 2012. Shaping networked theatre: experience architectures, behaviours and creative pedagogies. The J. Applied Theatre and Performance. (England). 17(4):603616. https://doi.org/10.1080/13569783.2012.727629

29. TAŞKIN-CAN, B. 2013. The Effects of Using Creative Drama in Science Education on Students' Achievements and Scientific Process Skills. Elementary Education Online. 12(1):120-131.

30. WAICHMAN, P. 2008. Tiempo Libre y Recreación, un desafío pedagógico. CCS, Madrid. 234p.

31. YOUNGS, I. 2013. La ciencia sube a escena en el mayor festival de teatro. Disponible desde Internet en: https://www.bbc.com/mundo/noticias/2013/08/130806_ ciencia_teatro_festival_fringe_edimburgo_np (con acceso 30/10/2018). 